\title{
ANÁLISIS COMPARATIVO DE SOFTWARE PARA OBTENER MDT CON FOTOGRAMETRÍA RPAS
}

\section{COMPARATIVE SOFTWARE ANALYSIS TO OBTAIN DTM WITH RPAS PHOTOGRAMMETRY}

\author{
Alba N. Arévalo-Verjela, ${ }^{a,}$, José Luis Lermaa ${ }^{a}$, José Fernández ${ }^{b}$ \\ ${ }^{a}$ Departamento de Ingeniería Cartográfica, Geodesia y Fotogrametría, Universitat Politècnica de València, Camino de Vera s/n, 46022 \\ Valencia, España. alarver@doctor.upv.es; jllerma@cgf.upv.es \\ b Instituto de Geociencias (IGEO), CSIC-UCM, Calle del Doctor Severo Ochoa 7, Ciudad Universitaria, 28040 Madrid, España. \\ jft@mat.ucm.es
}

\begin{abstract}
:
RPAS (Remotely Piloted Aircraft Systems) are widely used in photogrammetry for taking images due to their high spatial resolution and speed of response, being able to reach areas of difficult access, being important to design a good procedure in the field to minimize errors in data collection. It is recommended to use ground control points (GCP) using conventional RPAS, when they do not work with RTK (Real Time Kinematic) systems. Likewise, there are free and licensed photogrammetric programs on the market to generate digital surface models (DSM), terrain models (DTM) and orthophotomosaics. In this article, two photogrammetric programs are used to process images captured with RPAS, such as Agisoft Metashape and Recap Photo, using GCP and check points. The study was carried out in Almenara (Spain) where the topographic survey was carried out with RPAS, capturing 100 digital images, in an area of $0.38 \mathrm{~km} 2$. $6 \mathrm{GCP}$ were used in order to orient the digital images well in the local coordinate system and to properly georeference the images obtained during the flight. To obtain the MDT, the CloudCompare software was used to filter the cloud of points obtained from both software. The results show a difference in height between the two DTMs of less than $28 \mathrm{~cm}$, taking as a reference the DTM of the Agisoft Metashape point cloud and regarding the error in the check points, Recap Photo presented a greater error.
\end{abstract}

Key words: photogrammetry, RPAS, digital terrain model, GCP and check points, georeferencing

\section{Resumen:}

Los RPAS (Sistemas de aeronaves pilotados a distancia) son muy utilizados en fotogrametría para la toma de imágenes por su alta resolución espacial y rapidez de respuesta, pudiendo llegar a zonas de difícil acceso, siendo importante diseñar un buen procedimiento en campo para minimizar los errores en la toma de datos. Se recomienda utilizar puntos de apoyo (PA) terrestres utilizando RPAS convencional, que no trabajan con sistemas RTK (Real Time Kinematic). Asimismo, existen en el mercado programas fotogramétricos libres y licenciados para generar modelos digitales de superficie (MDS), del terreno (MDT) y ortofotomosáicos. En este artículo se utilizan dos programas fotogramétricos para procesar imágenes capturadas con RPAS como son Agisoft Metashape y Recap Photo, utilizando puntos de apoyo y control terrestre. El estudio se llevó a cabo en Almenara (España) donde se hizo el levantamiento topográfico con RPAS, capturándose 100 imágenes digitales, en un área de $0.38 \mathrm{~km}^{2}$. Se utilizaron 6 PA con la finalidad de orientar bien las imágenes digitales en el sistema de coordenadas local y realizar de forma adecuada la georreferenciación de las imágenes obtenidas durante el vuelo. Para la obtención del MDT se utilizó el software CloudCompare para hacer el filtrado en la nube de puntos obtenidas de ambos softwares. Los resultados muestran una diferencia en altura entre los dos MDT menor a $28 \mathrm{~cm}$ tomando como referencia el MDT de la nube de puntos de Agisoft metashape y en cuanto al error en los puntos apoyo Recap Photo presento mayor error.

Palabras clave: fotogrametría, dron, modelo digital del terreno, puntos de apoyo y de control, georreferenciación

\section{Introducción}

Las técnicas fotogramétricas son muy utilizadas para obtener información a partir de las fotografías o imágenes digitales, obtenidas por distintos sensores, (RPAS con cámaras fotográficas o fotogramétricas, cámaras $360^{\circ}$, teléfonos móviles, etc.). En la actualidad existen diversos programas, unos que requieren de licencia y otros de uso libre, capaces de procesar millones de puntos obtenidos mediante el procesamiento de estas fotografías.
Por otro lado con la fotogrametría con RPAS se pueden obtener modelos digitales del terreno (MDT) con algoritmos de filtrado, de manera similar a como se hace con las nubes de puntos LIDAR (Light Detection and Ranging), debido a sus características intrínsecas para clasificar puntos terrestres y no terrestres (Serifoglu Yilmaz and Gungor 2018), pero a un menor costo.

Los RPAS son muy utilizados en la actualidad por: i) ser uno de los sistemas que mejor resolución y nitidez presentan en la adquisición de imágenes utilizados en

*Corresponding Author: Alba N. Arévalo-Verjel, alarver@doctor.upv.es 
teledetección ii) la información espacial capturada es más densa que la registrada con trabajos de topografía clásica (Hernández 2006). Además los RPAS se utilizan para obtener imágenes aéreas en menor tiempo, y a diferentes alturas manteniendo una alta resolución para su uso en fotogrametría digital (Colomina and Molina 2014).

Para mejorar la precisión de los resultados obtenidos con RPAS es necesario el uso de herramientas adicionales como la estación total o el uso de GNSS (Sistema Global de Navegación por Satélite), nivelación (Jiménez et al. 2017). Para la validación en campo, se recurre a la implementación de PA (puntos de apoyo terrestres), y PC (puntos de control), logrando así generar productos fiables y comparables con la topografía convencional, pero con una densidad sensiblemente superior.

Los PA aumentan considerablemente la precisión del proyecto permitiendo desplazar el modelo a la posición exacta en el terreno (Agüera-Vega et al. 2017). Los PC sirven para cuantificar la precisión de los productos fotogramétricos obtenidos (Jiménez et al. 2017).

\section{Materiales y métodos}

La investigación se llevó a cabo en Almenara (España), donde se realizó el levantamiento topográfico con fotogrametría RPAS, siendo esta una técnica fotogramétrica utilizada para obtener MDT y ortomosaicos a partir de imágenes aéreas (Galeana et al. 2021). A una altura de vuelo de $120 \mathrm{~m}$, se capturaron 100 imágenes cubriendo un área de $0.38 \mathrm{~km} 2$, el modelo de la cámara usada es ILCE-5000 (16 mm) con una resolución de 5456 × 3632 y una distancia focal de $16 \mathrm{~mm}$ con un tamaño de pixel de $4.4 \times 4.4$ micras.

Para mejorar la precisión de los resultados se tomaron 6 PA distribuidos adecuadamente en el sitio de estudio, y 7 PC como se puede apreciar en la Figura 1.

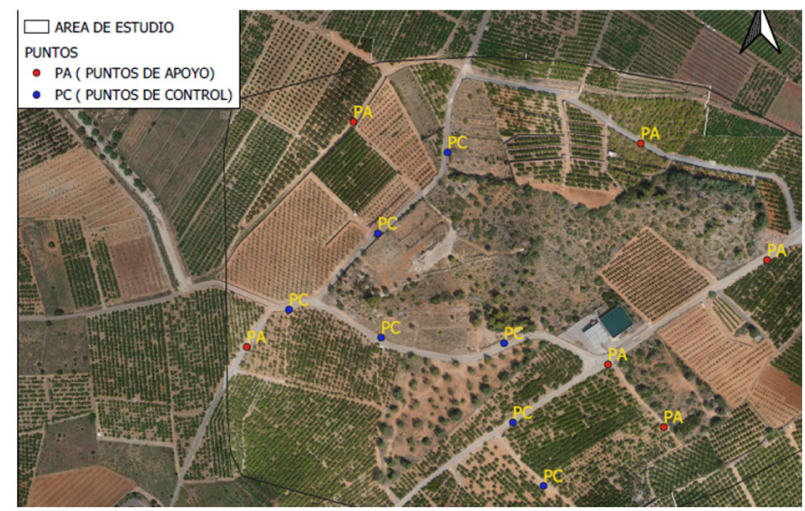

Figura 1: Distribución de puntos de apoyo $(\mathrm{PA})$ y de control (PC) en el área de estudio.

Para el procesamiento de datos se utilizó el software Agisoft Metashape y el software Recap Photo de Autodesk, con licencia educativa (Autodesk 2021).

El equipo utlizado es un lenovo procesador Intel $(R)$ core (TM) i5 -4210U CPU $1.70 \mathrm{GHz} 2.40 \mathrm{GHz}$, RAM $6 \mathrm{~GB}$, Windows 10 de 64 bits, tarjeta grafica NVIDIA GEFORCE $820 \mathrm{M}$.

\subsection{Procesamiento mediante Agisoft Metashape}

El software Agisoft Metashape realiza el procesado fotogramétrico de imágenes digitales y genera datos espaciales en 3D que pueden ser utilizados en aplicaciones SIG (Sistemas de Información Geográfica).

Antes de iniciar con el proceso en Agisoft se debe activar en preferencias la opción de GPU (Unidad de Procesamiento Gráfico), para mejorar la velocidad de procesamiento, dado que el software no la detecta automáticamente. A continuación, se sigue el flujo de trabajo que se indica en la Figura 2.

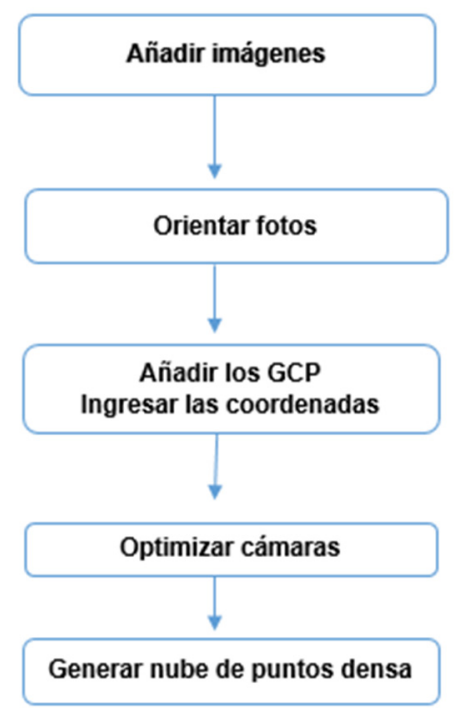

Figura 2: Flujo de trabajo Agisoft Metashape.

Para dar continuidad al proceso se deben ingresar las coordenadas de los GCP, manualmente o importando un archivo como fichero txt, y configurar el proyecto en el mismo sistema de coordenadas. Adicionalmente se activa la opción de optimizar cámaras para que toda la nube de puntos sea corregida (Alexander and Acurio 2018). Se dejan activos solo los PA, que para este proyecto son los puntos 87, 89, 90, 95, 105, 107 (Fig. 3). Se busca que la distribución de los puntos queden en lados opuestos para lograr una mejor georreferenciación y los puntos restantes quedan como PC.

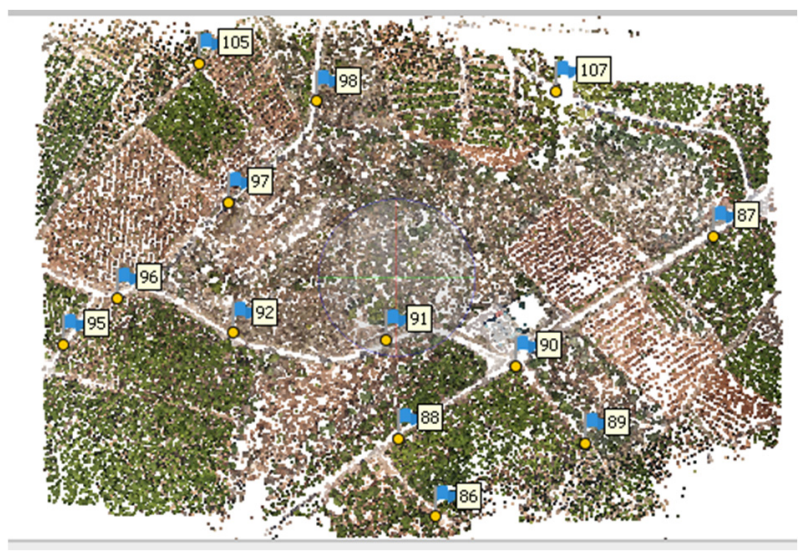

Figura 3: Localización de los PA y PC. 
En el flujo de trabajo se procede a crear la nube de puntos densa. Con base a esta se procede a generar el MDT y ortomosaico.

Para generar el MDT se usa la opción de filtrado de puntos con la opción de herramientas - nube de puntos densa - clasificación del terreno, que debe ser estudiado para ver la calidad de este proceso.

\subsection{Proceso mediante Recap Photo}

Recap photo es un servicio incluido con Recap Pro, que procesa fotografías de RPAS para crear representaciones 3D. También admite la creación de nubes de puntos, mallas y ortofotos (Autodesk 2021).

El procedimiento en este software es similar al de Agisoft: primero se usa el tipo de datos aerial para el caso de las fotografías con RPAS, el flujo de trabajo se muestra en la Figura 4.

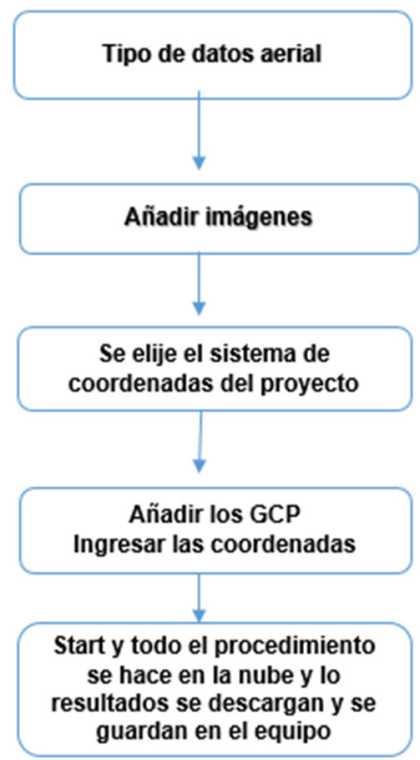

Figura 4: Flujo de trabajo en Recap Photo.

Después de ingresar las fotos se introducen los PA y PC como se muestra en la Figura 5 . Estos pueden ser importados desde un archivo txt, después se activa la herramienta filtrado por GNSS para que muestre las imágenes para cada punto, que debe ser marcado como mínimo en tres fotografías diferentes. Después de esto se procesa y se guarda el reporte en el equipo de trabajo, de lo contrario quedará almacenado en la nube del software.

\section{Resultados}

EI MDT, modelo digital de elevación que describe la superficie de terreno, es un conjunto de datos de dimensión 2.5, es decir, extrapolan la coordenada $Z$ a partir de las coordenadas planimétricas $X Y, Z=f(X, Y)$. Parar cada punto $X Y$ sólo existe una $Z$ (Lerma 2002). Son muy utilizados en ingeniería para el cálculo de volúmenes, perfiles longitudinales, medir asentamientos del terreno entre otros.

Actualmente los MDT se pueden generar a través de varias técnicas que incluyen principalmente el radar de apertura sintética interferométrica (InSAR), la detección y determinación de distancia de la luz en el aire (LiDAR) y la fotogrametría digital basada en imágenes aéreas y satelitales (Chen et al. 2016), y la presentada en este articulo utilizando fotografías tomadas con RPAS.

Los MDS y el ortomosaico es la etapa final del procesamiento de imágenes obtenidas con RPAS. Para la generación de estos modelos la nube de puntos debe pasar por una triangulación y rasterización (Escalante and Porras 2016). Para la generación del ortomosaico las imágenes individuales son rectificadas a partir del MDT, eliminando la distorsión asociada al relieve.

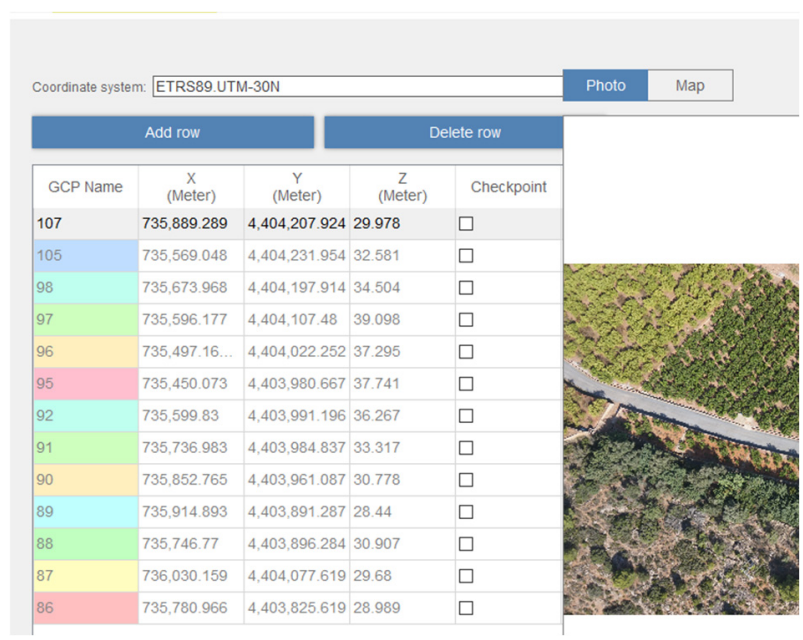

Figura 5: Ingreso de PA y PC en el software Recap Photo.

\subsection{Resultados con Agisoft Metashape}

EI GSD (Ground sampling distance) que indica el tamaño aproximado que representa un pixel en el terreno (Quispe 2015). Para este proyecto es de $3.67 \mathrm{~cm} /$ pix., que coincide con el teórico, indicando que el vuelo se mantuvo a una altura constante.

Es importante desmarcar los PC cuando se generen los modelos para que el programa no procese todos los puntos como PA. En la Tabla 1 se presentan los errores obtenidos para los $6 \mathrm{PA}$, generando un error total en $X$ de $0.29 \mathrm{~cm}$, en $Y$ de $0.68 \mathrm{~cm}$, y un error en $Z$ de $1.12 \mathrm{~cm}$ que es el que más interesa para validar el MDT, dando muy buena precisión en los PA, porque los errores son menores a los $2 \mathrm{~cm}$.

Tabla 1: Error en los PA.

\begin{tabular}{c|c|c|c|c} 
Punto & $\begin{array}{c}\text { Error } X \\
(\mathrm{~cm})\end{array}$ & $\begin{array}{c}\text { Error } Y \\
(\mathrm{~cm})\end{array}$ & $\begin{array}{c}\text { Error Z } \\
(\mathrm{cm})\end{array}$ & $\begin{array}{c}\text { Total } \\
(\mathrm{cm})\end{array}$ \\
\hline 90 & -0.2 & -1.4 & 1.1 & 1.8 \\
95 & 0.6 & 0.1 & 0.5 & 0.8 \\
89 & -0.1 & 0.8 & -1.7 & 1.9 \\
87 & -0.2 & 0.0 & 1.6 & 1.6 \\
107 & 0.1 & 0.3 & -0.7 & 0.8 \\
105 & -0.3 & 0.3 & -0.3 & 0.5 \\
Total & 0.3 & 0.7 & 1.1 & 1.3
\end{tabular}

\subsubsection{Modelo digital del terreno (MDT)}

El MDS que se generó en Agisoft se obtuvo con una resolución de $6.71 \mathrm{~cm} /$ pix y una densidad de puntos de 
222 puntos $/ \mathrm{m} 2$ con un valor de altura mínima de $22 \mathrm{~m}$ y máximo de $52 \mathrm{~m}$ como se observa en la Figura 6 . Dentro de la etapa de procesamiento de las imágenes, la discretización de la nube de puntos densa en tres clases es un paso fundamental para obtener los modelos que representan la topografía del sitio de estudio. La discretización permite triangular únicamente los puntos de terreno para generar los MDT (Jiménez et al. 2017). Hay que clasificar la nube puntos en puntos de ruido (valores atípicos por encima y debajo del suelo), objetos y puntos del terreno.

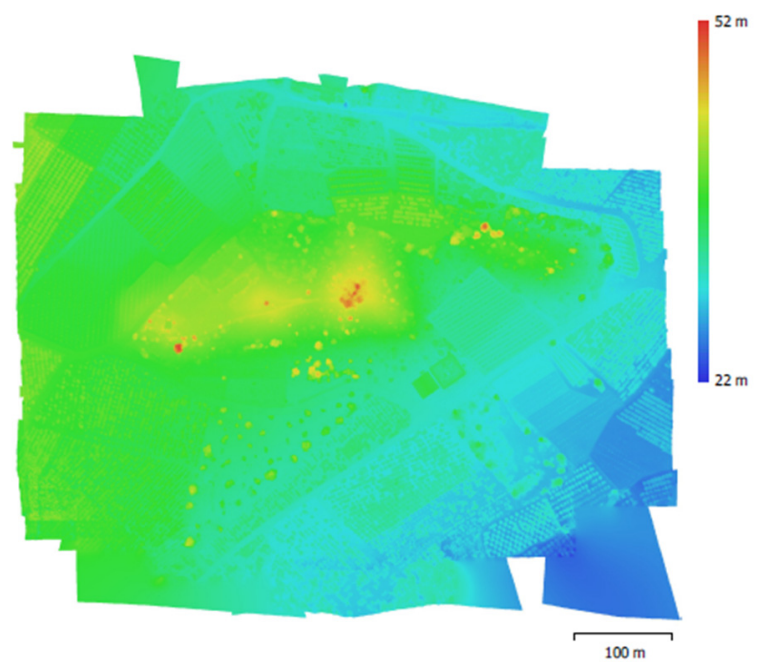

Figura 6: Raster de MDS software Agisoft Metashape.

Para convertir el MDS en MDT el software cuenta con una herramienta a partir de la nube de puntos densa y con la opción de clasificar puntos en terreno como se observa en la Figura 7. También se utilizo el visor de nubes de puntos ColudCompare para clasificar solo los puntos que son del terreno.

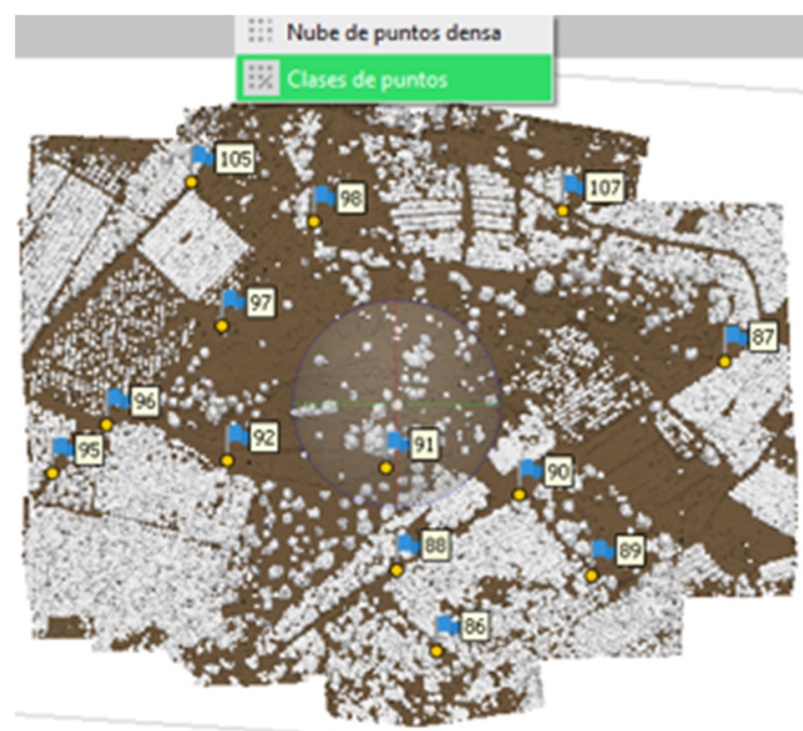

Figura 7: Clasificación de puntos software Agisoft Metashape.

Analizando los resultados con CloudCompare utilizando la nube de puntos generada por el software Agisoft Metashape, se creo un campo escalar con la coordenada $Z$ para la nube de puntos. Posteriormente, se ajustó el histograma resultante. La Figura 8 muestra un valor mínimo de 24 y un máximo de $48 \mathrm{~m}$.

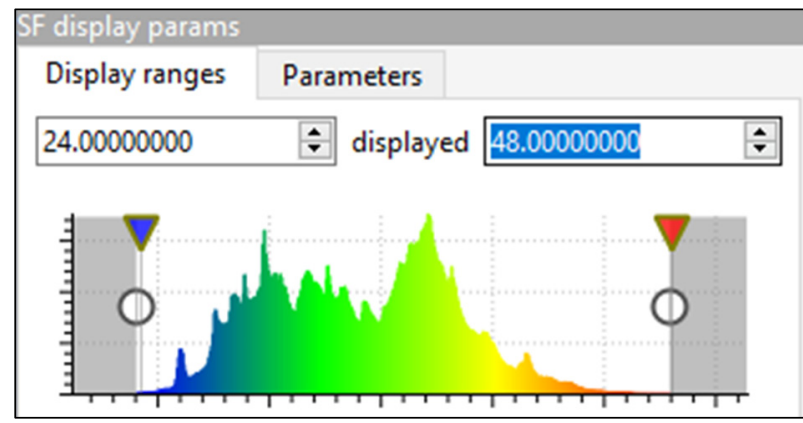

Figura 8: Mejora del histograma de la nube de puntos generada con Agisoft Metashape en CloudCompare.

En la Figura 9 se muestra los resultados después de ajustar el histrograma a la nube de puntos con el nuevo campo escalar $Z$ generado. Se puede comprobar que se aprecia mejor el contenido del MDS.

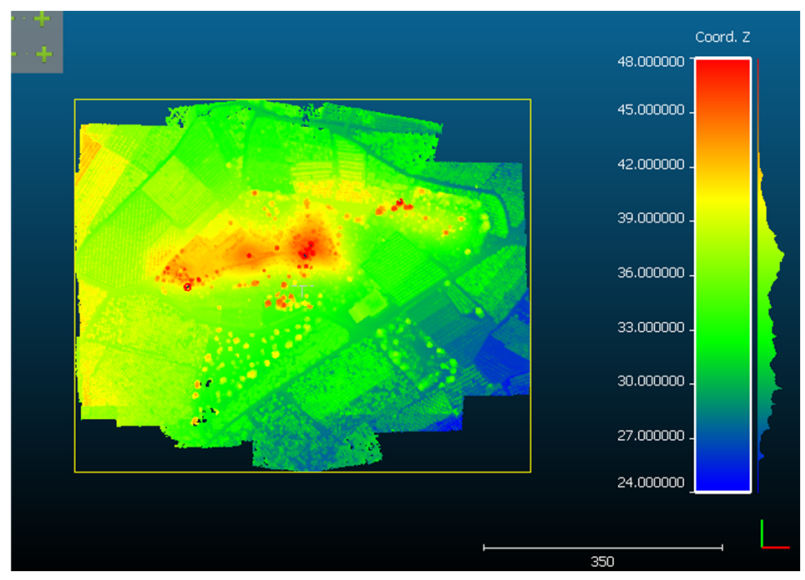

Figura 9: Resultado de mejorar el contraste del campo escalar $Z$ en CloudCompare.

Para realizar el filtrado de puntos en CloudCompare se utilizó la herramienta CSF filter (siglas en inglés de Cloth Simulation Filter), que permite clasificar la nube de puntos en nube de puntos terreno y no terreno (Fig. 10). Se escogió la opción de relieve porque el terreno no es plano, procesado con pendiente, y se repitió este proceso tres veces hasta que la nube quedara solo con puntos pertenecientes al terreno. Con la herramienta de Edit $\rightarrow$ Merge se fusionaron las tres nubes de puntos ya filtradas. Los resultados se muestran en la Figura 10.

Los resultados los podemos exportar en formato ráster. ASC II, las, laz, PLY, DXF etc.

\subsection{Resultados con el software Recap Photo}

Recap Photo se encuentra dentro de la suscripción de Recap pro, es una aplicación conectada a la nube, generando la ortofoto y un MDS como se muestra en la Figura 11, dando como resultado una resolución de 10 $\mathrm{cm} /$ pix para una altura mínima de $22.34 \mathrm{~m}$ y máxima de $51.22 \mathrm{~m}$. Comparándolo con el resultado que genero Agisoft, estos se asemejan ya que cuando se genera el reporte en este software se aproximan las alturas, aunque presentó un cambio en la resolución. En cuanto 
al tiempo necesario para el procesado, este depende de cuantos proyectos estén en cola en la nube, si usamos licencia educativa este proceso tardara mas y para procesar mas de 100 fotografias hay que adquirir la licencia, el tiempo que tardo este proyecto fue de 8 horas.

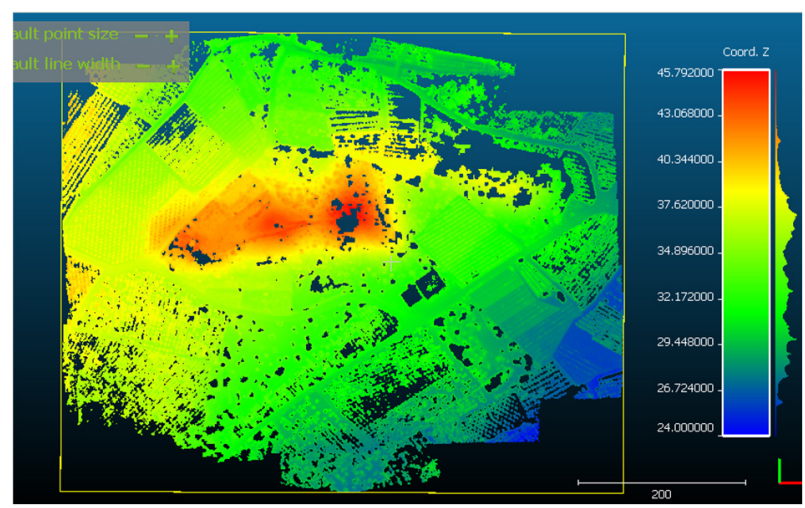

Figura 10: Filtrado de puntos de la nube generada por Agisoft Metashape utlilizando CSF Filter de Cloud Compare.

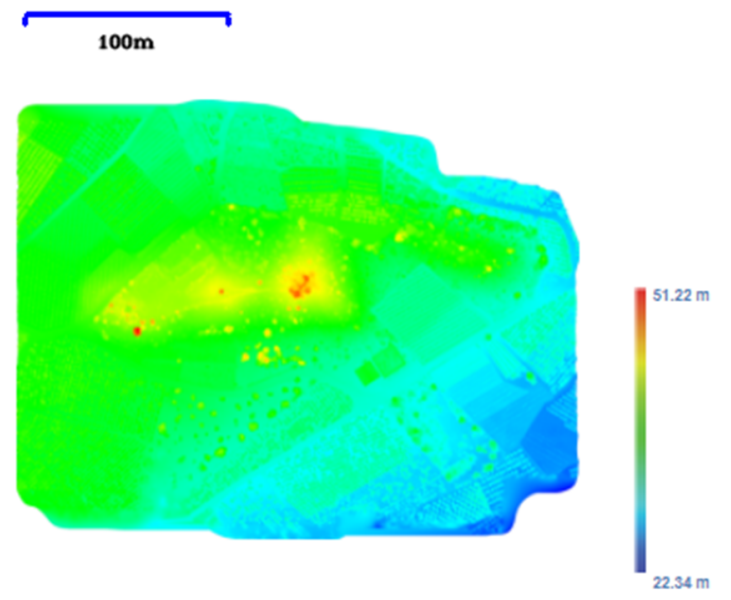

Figura 11: Digital Elevation Model. Software Recap Photo (MDS).

En cuanto a los PA, el error es menor de $6.7 \mathrm{~cm}$ en $\mathrm{XYZ}$ como se observa en la Tabla 2. Estos son calculados por las diferencias entre las entradas del usuario (precisión al ubicar el punto en el software) y los puntos de coordenadas calculadas por el sistema, y está relacionado con el GSD los valores $X, Y$ y $Z$ de la tabla corresponden al sistema de coordenadas geográficas.

Tabla 2: Error en los PA.

\begin{tabular}{c|c|c|c|c|c} 
Pto & $\begin{array}{c}X \\
\text { (latitud) }\end{array}$ & $\begin{array}{c}Y \\
\text { (longitud) }\end{array}$ & $\begin{array}{c}Z \\
\text { (altitud) }\end{array}$ & $\begin{array}{c}\text { Error } X Y \\
(m)^{*}\end{array}$ & $\begin{array}{c}\text { Error } Z \\
(m)^{*}\end{array}$ \\
\hline 95 & -0.25 & 39.7 & 37.74 & $<0.067$ & $<0.067$ \\
90 & -0.24 & 39.7 & 30.78 & $<0.067$ & $<0.067$ \\
89 & -0.24 & 39.7 & 28.45 & $<0.067$ & $<0.067$ \\
87 & -0.24 & 39.7 & 29.68 & $<0.067$ & $<0.067$ \\
105 & -0.25 & 39.7 & 32.58 & $<0.067$ & $<0.067$ \\
107 & -0.24 & 39.7 & 29.98 & $<0.067$ & $<0.067$
\end{tabular}

Para convertir el MDS en MDT se exportó la nube de puntos en formato $\mathrm{X}, \mathrm{Y}, \mathrm{Z}$ para ser analizada en CloudCompare y se realizó el mismo procedimiento que se manejo para la nube de puntos generada por Agisoft Metashape. Los resultados se muestran en la Figura 12.

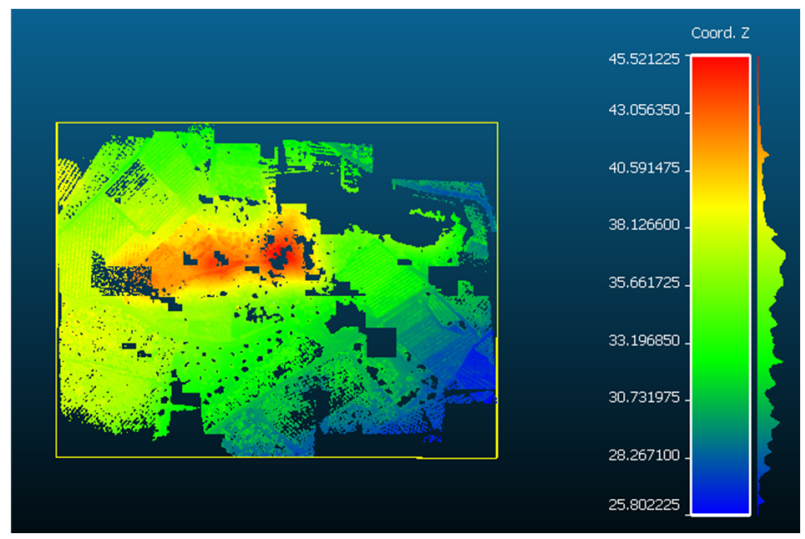

Figura 12: Filtrado de puntos de la nube generada por Recap Photo utlilizando CSF Filter de CloudCompare.

\section{Discusión}

Los resultados de las dos nubes de puntos obtenidas después de clasificar solo los puntos del terreno son analizados con la herramienta compute cloud distance de CloudCompare que busca el punto más cercano en la nube de referencia y calcula su distancia euclidea.

Tomando como referencia la nube de puntos generada por Agisoft Metashape y comparándola con la nube de Recap Photo, y utilizando la herramienta tools-statisticscompute-stats-params se obtuvieron los resultados mostrados en la Figura 13.

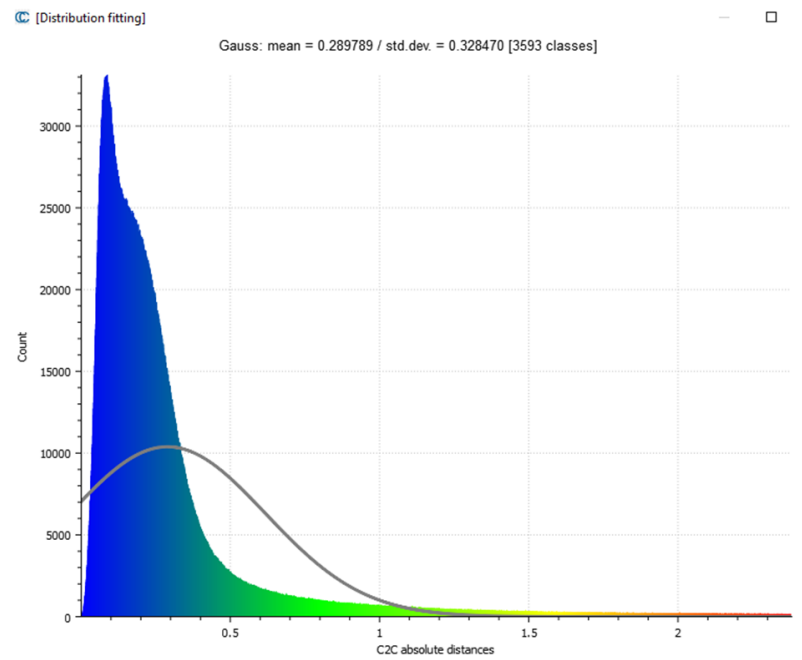

Figura 13: Comparación entre las nubes de puntos con la campana de Gauss.

De la Figura 13, el eje de la abscisa representa la distancia en metros entre los dos modelos y el de la ordenada representa la cantidad de puntos, podemos observar que la media de las distancias calculadas entre las nubes de puntos es de $28 \mathrm{~cm}$, con una desviación estándar de $32 \mathrm{~cm}$. Estos valores no siguen una distribución normal, y la mayoría de puntos entre los dos modelos se encuentran por debajo de los $28 \mathrm{~cm}$.

En cuanto a la densidad de puntos el MDT de la nube de puntos generada en Metashape y filtrada en CloudCompare es de $51 \mathrm{ppm}^{2}$ con una altura minima de 
$24 \mathrm{~m}$ y la máxima $45.792 \mathrm{~m}$; para la nube de Photo obtenemos una densidad de $47 \mathrm{ppm}^{2}$ una altura minina de $25.8 \mathrm{~m}$ y una máxima de $45.52 \mathrm{~m}$.

En la Tabla 3 se presentan en resumen las diferencias en cuanto al tiempo de procesado de las imágenes y errores en los PA de los dos softwares utilizados. Es necesario aclarar que el tiempo en Agisoft varia en función de las características de hadware utilizado.

Tabla 3: Comparativa entre Agisoft metashape y Recap Photo.

\begin{tabular}{c|c|c} 
Caracteristicas & $\begin{array}{c}\text { Agisoft } \\
\text { Metashape }\end{array}$ & $\begin{array}{c}\text { Recap } \\
\text { Photo }\end{array}$ \\
\hline Tiempo de procesado $(\mathrm{h})$ & 5 & 8 \\
Error total en Z en los PA $(\mathrm{cm})$ & 1.1 & $<6.7$
\end{tabular}

El tiempo en Recap Photo depende de los proyectos que estén en cola dependientdo el tipo de licencia que se utilize, debido a que este procesa en la nube.

\section{Conclusiones}

En la actualidad existen diferentes tipos de programas capaces de realizar todas las funciones de procesado necesarias para generación de ortomosaicos y MDT. La diferencia entre Agisoft y Recap Photo, es que recap procesa todo en la nube y no se sabe cuanto tiempo pueda durar el procesado, y además no se puede ir ajustando los parámetros de procesado, mientras que en Agisoft se puede ir ajustando al tiempo y las necesidades del proyecto.
Para generar un MDT Agisoft Metashape tiene la herramienta para filtrar solo los puntos del terreno pero la calidad no es muy óptima porque se pueden apreciar los árboles en el modelo. El software Recap Photo da un resultado similar al de Agisoft Metashape en cuanto al MDS pero para el filtrado de puntos que no son del terreno, se requiere del software Civil $3 D$ para realizar este procedimiento, porque dentro de Recap Photo no existe la opción. Otra alternativa para utilizar este software es extraer la nube de puntos en formato OBJ o como XYZ para poder hacer el filtrado del MDS a MDT, por ejemplo, en CloudCompare. En cuanto a exportar archivos, Agisoft Metashape tiene multiples opciones de formatos de salida, y Recap Photo es más limitado.

EI MDT resultado de Agisoft presenta mayor densidad en la nube de puntos que el resultado en la nube de puntos de Recap Photo. En cuanto a la calidad del MDT los dos softwares presentan una diferencia menor a los $28 \mathrm{~cm}$.

Ambos softwares, Agisoft Metashape y Recap Photo, son muy eficientes para el procesado de imágenes y creación de ortomosaicos, pero para generar MDT se deben complementar con otros softwares que tienen implementados mejores procesos de filtrado, como son CloudCompare o LAStools.

\section{Agradecimientos}

Se agradece a Autodesk por la licencia otorgada por un año de todos sus programas con fines académicos.

Esta investigación se ha sufragado parcialmente por el proyecto de la AEI DEEP-MAPS (RTI2018-093874-B100).

\section{References}

AGÜERA-VEGA, F., CARVAJAL-RAMÍREZ, F., and MARTÍNEZ-CARRICONDO, P., $2017 . \quad$ Assessment of photogrammetric mapping accuracy based on variation ground control points number using unmanned aerial vehicle. Measurement: Journal of the International Measurement Confederation, 98, pp. 221-227. DOI: 10.1016/j.measurement.2016.12.002

AUTODESK, INC. 2021. Software de Recap. Available: https://www.autodesk.es/products/recap/overview?term=1-YEAR $[8 / 22,2021]$

ALEXANDER, G., and ACURIO, C., 2018. Análisis comparativo entre los software de prueba agisoft photoscan y pix4d para el procesamiento de datos obtenidos con fotogrametría de vehículo aéreo no tripulado (uav) de bajo costo. Trabajo de grado, Escuela Politécnica Nacional, Quito, Ecuador.

CHEN, Q., WANG, H., ZHANG, H., SUN, M., and LIU, X., 2016. A point cloud filtering approach to generating DTMs for steep mountainous areas and adjacent residential areas. Remote Sensing, 8(1). DOI: 10.3390/rs8010071

COLOMINA, I., and MOLINA, P., 2014. Unmanned aerial systems for photogrammetry and remote sensing: A review. ISPRS Journal of Photogrammetry and Remote Sensing, 92, pp. 79-97. DOI: 10.1016/J.ISPRSJPRS.2014.02.013

GALEANA, V. M., CHÁVEZ, O., and MEDELLÍN, G., 2021. On the measure of land subsidence throughout DEM and orthomosaics using GPS and UAV. Ingeniería Investigación y Tecnología, 22(1), pp. 1-12. DOI: 10.22201/fi.25940732e.2021.22.1.006

HERNÁNDEZ, D., 2006. Introducción a la fotogrametría digital. ETSI Agrónomos: Universidad de Castilla la Mancha.

JIMÉNEZ-JIMÉNEZ, S., OJEDA-BUSTAMANTE, W., ONTIVEROS-CAPURATA, E., FLORES-VELÁZQUEZ, J., MARCIAL-PABLO, M DE J., BRAULIO, and ROBLES-RUBIO, D., 2017. Quantification of the error of digital terrain models derived from images acquired with UAV. Ingeniería agrícola y biosistemas, 9(2), pp. 85-100.DOI: 10.5154/r.inagbi.2017.03.007

LERMA, J. L., 2002. Fotogrametria Moderna: Analitica y Digital. Universidad Politécnica de Valencia, pp. 556. ISBN: 97884-9705-210-8

QUISPE, O. C., 2015. Análisis de GSD para la generación de cartografía utilizando la tecnología drone. Huaca de la Universidad Nacional Mayor de San Marcos, 18. 
SERIFOGLU YILMAZ, C., and GUNGOR, O., 2018. Comparison of the performances of ground filtering algorithms and DTM generation from a UAV-based point cloud. Geocarto International, 33(5), pp. 522-537. DOI: 10.1080/10106049.2016.1265599

ESCALANTE, J. O., and PORRAS, H., 2016. Orthomosaics and digital elevation models generated from images taken with UAV systems. Tecnura, 20(50), pp. 119-140. DOI: 10.14483/22487638.11566 